\title{
Broncoscopia intervencionista terapéutica en pediatría
}

\section{Therapeutic interventional bronchoscopy in pediatrics}

\author{
Oscar Barón Puentes, MD.
}

\section{Resumen}

Gracias al desarrollo de equipos adecuados para la realización de estudios broncoscópicos en niños este procedimiento se ha ido posicionando como un estudio común y esencial en el manejo de diversas patologías respiratorias en pediatría. La broncoscopia ha demostrado ser útil en la remoción de cuerpos extraños impactados, la aspiración guiada de tapones de moco y coágulos retenidos en los bronquios, la asistencia en intubaciones difíciles, en dilataciones de estenosis de tráquea o bronquios congénitas o adquiridas, en ablación de lesiones de la mucosa, aspiración transtraqueal con aguja fina de quistes y nódulos, lavado pulmonar ante sospecha de infección, cierre de fístulas broncopleurales y en diversas aplicaciones perioperatorias como intubaciones selectivas y cierre de fístulas traqueoesofágicas. Es necesaria la creación de más centros especializados en Colombia que cuenten con tecnología de punta y personal capacitado para cambiar el pronóstico de muchos problemas respiratorios serios en la población pediátrica.

Palabras clave: broncoscopia pediátrica, estenosis de la vía aérea, cuerpo extraño, intubación difícil, neumología pediátrica.

\begin{abstract}
Thanks to the development of suitable equipment for bronchoscopic studies in children, this procedure has positioned itself as a common and essential test in the management of diverse respiratory diseases in pediatrics. Bronchoscopy has proved to be useful for removal of impacted foreign bodies, guided aspiration of plugged up mucus and blood clots in the bronchi, assistance in difficult intubation, dilatation of congenital or acquired tracheal or bronchial stenosis, resection of mucosal lesions, transtracheal needle aspiration of cysts and nodules, pulmonary lavage in cases of suspected infection, closure of bronchopleural fistulas, and various perioperative applications, such as selective intubation and closure of tracheoesophageal fistulas. In Colombia, it is necessary to create more specialized centers with leading-edge technology and proficient staff in order to change the prognosis of many serious respiratory problems in the pediatric population.
\end{abstract}

Keywords: pediatric bronchoscopy, airway stenosis, foreign body, difficult intubation, pediatric pulmonology.

\section{Introducción}

Desde 1980, año en que se dispuso de equipos adecuados para realizar estudios broncoscópicos en niños, la broncoscopia ha ido posicionándose como un elemento diagnóstico de primera línea, hasta ser considerada hoy como relevante y necesaria para el manejo de un grupo importante de enfermedades respiratorias del niño. Sin embargo, su aplicación en nuestro medio ha venido limitándose, en la mayoría de los casos, a la simple inspección del aparato respiratorio, con fines puramente diagnósticos.

\footnotetext{
(1)Neumólogo Pediatra. Profesor Clínico Universidad de la Sabana, Universidad El Rosario. Coordinador Servicio de Broncoscopia Pediátrica, Fundación Neumológica Colombiana. Bogotá, Colombia.

Correspondencia: Oscar Barón Puentes. Correo electrónico: obaron@cardioinfantil.org Recibido: 16/10/14, Aceptado: 10/12/14.
} 
Se utiliza solo en un pequeño grupo de patologías poco comunes, mientras que apenas en algunos casos, más seleccionados, se complementa con la realización del lavado broncoalveolar como un método diagnóstico más específico con el que se busca encontrar la etiología de procesos generalmente inflamatorios e infecciosos que ocurren en el tracto respiratorio del menor.

En contraste con la realidad colombiana, en años recientes, en el mundo "desarrollado", ha habido avances considerables en cuanto a técnicas y tecnología que permiten dar solución a múltiples patologías que antes no tenían tratamiento o para las que era necesario recurrir a procedimientos muy invasivos y traumáticos con resultados no siempre exitosos. Con estos avances hoy se pueden abordar y solucionar múltiples alteraciones y patologías de la vía aérea. Con su realización en sitios seleccionados y a cargo de personal médico debidamente entrenado, se obtienen resultados exitosos y altamente seguros para el paciente pediátrico.

\section{¿Por qué puede ser necesario realizar broncoscopia intervencionista en niños?}

En 1897, Kilian hizo una extracción de un fragmento de hueso de cerdo del bronquio de un paciente, utilizando un tubo rígido iluminado con una fuente de luz indirecta. Se considera que la historia de la broncoscopia intervencionista, se inició a partir de ese ya lejano momento. Desde entonces, los progresos han sido inexorables. Por ejemplo, el diseño de tubos endoscópicos más delgados desarrollados por Chevalier Jackson a principios del siglo 20, permitieron, por primera vez en la historia, efectuar procedimientos en niños. De más tarde data la aparición del fibrobroncoscopio flexible ideado por Ikeda en Japón en 1967, y el desarrollo en 1980 de fibrobroncoscopios pediátricos diseñados por Robert Wood, broncólogo estadounidense considerado el pionero en su uso en Pediatría y quien estableció las primeras indicaciones. Vale la pena relatar cómo el propio Wood cuenta que en su primera presentación sobre los hallazgos endoscópicos realizados en niños, fue tildado de "voyerista médico" y de mala praxis profesional. Por fortuna esto ha sido superado y hoy la broncoscopia goza de gran prestigio, ganado por su innegable seguridad y eficacia. Todos estos avances han permitido, además, la aparición de una nueva subespecialidad pediátrica (broncoscopia pediátrica) que es aplicable tanto en la clínica diaria como en la investigación de enfermedades respiratorias. Es claro que con el advenimiento de equipos cada vez más perfectos en cuanto a iluminación, tamaño y resolución, además de la aparición de aparatos e instrumental adicionales, ha ido ganándose en precisión, seguridad y eficiencia.

Por supuesto, las indicaciones y los métodos de la broncoscopia pediátrica difieren de los de los adultos; en estos últimos predomina el cáncer, con una expectativa de vida corta, mientras que en los niños la patología congénita y las anomalías estructurales adquiridas, dominan el panorama. Aunque hay aún alguna limitación en cuanto a experiencia y equipos apropiados y se requieren aproximaciones multidisciplinarias, el procedimiento sigue superando el balance riesgo-beneficio en contraposición con abordajes quirúrgicos complejos y riesgosos, como la traqueostomía y el soporte ventilatorio prolongado o simplemente cuando solo se limita al manejo de soporte básico o expectante, con la única esperanza de que algún día haya mejoría producto del mero desarrollo natural del niño.

Obviamente, la broncoscopia intervencionista es terapéutica más que solo diagnóstica, y su objetivo primario es restaurar la permeabilidad o patencia de la vía aérea. Ha demostrado ser útil en la remoción de cuerpos extraños impactados, aspiración guiada de tapones de moco y coágulos retenidos en los bronquios, asistencia en intubaciones donde la exposición de la laringe es difícil o crítica, dilataciones de estenosis de tráquea o bronquios, ablación de lesiones en la mucosa con láser o argón plasma, aspiración transtraqueal con aguja fina de quistes y nódulos, lavado pulmonar 
total, cierre endoscópico de fístulas broncopleurales, broncoscopia perioperatoria, como por ejemplo acompañamiento en cirugías complejas de tórax, intubaciones selectivas y cierre de fístulas traqueoesofágicas, entre otros, es decir su utilidad es amplia en cuanto a patologías y casi infinita en lo concerniente a posibilidades terapéuticas.

\section{Condiciones necesarias para la realización de broncoscopia intervencionista}

La justificación para la realización de broncoscopia depende, en principio, del interrogante que se desea resolver. Debe hacerse siempre bajo una estricta planeación con el equipo multidisciplinario que interviene en el procedimiento, es decir anestesiólogos experimentados, instrumentadoras o auxiliares, ya que los riesgos de hacerla o no dependen de la información buscada, y en última instancia, debe pensarse que si la broncoscopia tiene el potencial de resolver la duda, entonces, los beneficios generalmente superarán los riesgos.

Ante la duda de algunos médicos que se preguntan si realmente existe broncoscopia terapéutica en niños, la respuesta es enfática, sí. Ya se mencionaron algunas indicaciones, pero ahora también existe la posibilidad de instilar de manera dirigida medicamentos como dornasa alfa para licuar tapones de moco, en especial en pacientes con fibrosis quística y otros tipos de bronquiectasias severas; además, se pueden implantar endoprótesis (stents) bronquiales y realizar terapia endobronquial con láser o terapia endobronquial con ayuda de imágenes tridimensionales.

En unidades neonatales y de cuidado intensivo donde el paciente permanece intubado, es más sencillo decidir si se hace el procedimiento, en cuyo caso puede estar indicada en un 44 a $90 \%$ de los pacientes. Se convierte incluso en una oportunidad para resolver situaciones específicas como atelectasias persistentes, extracción de tapones de moco o evaluación de la vía aérea antes de una extubación, o también para el estudio de infecciones oportunistas o nosocomiales.
En el caso del diagnóstico de las enfermedades intersticiales, si bien no hay aún un consenso unánime, se considera que la biopsia transbronquial, ante todo en las enfermedades difusas, puede ser un paso intermedio antes decidir realizar la biopsia abierta, que parece ser el estándar pero que no está exenta de riesgos, especialmente en este grupo de niños que están muy comprometidos desde el punto de vista clínico.

En la tabla 1 se enumeran las indicaciones más frecuentes de esta intervención:

Tabla 1. Indicaciones generales de la broncoscopia intervencionista (terapéutica) en Pediatría

- Lesiones estenóticas de la vía aérea
- Malformaciones congénitas (malacias, estenosis)
- Lesiones post-infecciosas o secuelares
- Aplicación de medicamentos
- Remoción de tapones o impactaciones mucoides
- Extracción de cuerpos extraños
- Intubaciones difíciles o selectivas

\section{Manejo broncoscópico de las estenosis de la vía aérea en pediatría}

\section{Evaluación endoscópica}

El primer paso para decidir la intervención es definir el calibre y grado de estrechez de la vía aérea, y para ello se han diseñado bujías elásticas de goma (Nelaton), las cuales sirven para medir el calibre y la longitud de la estenosis previo a la intervención. En algunos lugares se han usado como método definitivo para dilatar la vía aérea aplicando de manera secuencial bujías de calibre creciente hasta el diámetro final deseado con aplicación de mitomicina tópica al final para prevenir recurrencias. Esta técnica es efectiva pero no 
exenta de riesgos (desgarros, ruptura bronquial, hemorragia), por lo cual muchos broncoscopistas no la recomiendan como procedimiento de rutina.

\section{Reparación de estenosis}

La siguiente es una guía general para la elección del método más adecuado:

a. Lesiones mucosas de consistencia blanda: se tratan mejor con fotorresección (ej. lesiones en forma de anillo, granulomas, quistes mucoides y tumoraciones).

b. Traqueobroncomalacias segmentarias, compresiones extrínsecas o lesiones por rotación o torsión del árbol bronquial: se pueden tratar mejor con stents, como alternativa a cirugía o traqueostomía.

c. Lesiones rígidas fibrocartilaginosas: pueden dilatarde mediante broncoscopio rígido (ej. anillos cartilaginosos congénitos, estrecheces isquémicas y estenosis anastomóticas).

d. Lesiones complejas: pueden requerir una combinación de las anteriores (ej. fibrorresección de lesiones mucosas más aplicación de endoprótesis, o aplicación de endoprótesis del segmento colapsado inducido por dilatación endoscópica de una estenosis fija).

\section{Técnicas}

\section{Dilatación neumática endoluminal}

Es el método más usado para tratar las estenosis traqueobronquiales adquiridas y se debe usar antes que otros métodos más invasivos. Se basa en la aplicación de altas presiones usando un balón que es llenado con solución salina o agua. Se hace bajo control visual mediante una lente óptica que se pasa a través del broncoscopio, habiendo evaluado y localizado previamente el área estenótica a través de imágenes diagnósticas, sea por tomografía computarizada o por broncoscopia virtual. Es útil en estrecheces fibrocartilaginosas y en otras estrecheces.

\section{Dilatación con tubos rígidos}

Es un tratamiento prequirúrgico para anillos cartilaginosos completos mediante visión por fluoroscopia, pero infortunadamente, no es muy recomendable pues hay experiencia limitada en este tipo de procedimiento.

\section{Fotorresección con láser}

Puede usarse en múltiples tipos de lesiones, principalmente iatrogénicas, como estenosis subglóticas o granulomas supraostomales.

La principal indicación es la escisión de granulomas previo a la extubación. Otras son: eliminación de anillos completos, marsupialización de quistes traqueobronquiales y sellado de fístulas traqueosofágicas pequeñas. No se recomienda para tumores que obstruyen la luz, pues pueden empeorar, a excepción del carcinoide, que responde muy bien. En tuberculosis primaria con nódulos y gran compromiso endobronquial, se usa cuando la administración de quimioterapia y la corticoterapia no han funcionado. El tipo de láser ideal en niños es el Dye Laser pulsado, por ser más efectivo y seguro.

\section{Endoprotesis (stents) traqueobronquiales}

Son aparatos protésicos para mantener la integridad de estructuras tubulares huecas. En términos genéricos existen dos tipos de stents, los de silicona y los metálicos o coils. Ambos pueden ser insertados por vía broncoscópica. Se han usado para el manejo de traqueobroncomalacias, torsiones o compresiones de la vía aérea por cirugía cardiotorácica, estenosis de anastomosis posttransplante y estenosis adquiridas o congénitas.

La traqueobroncomalacia en niños es la indicación más frecuente, aunque aún se usa la aortopexia.

Cuando la cirugía es técnicamente imposible o inviable, la colocación de stents es la mejor opción, aunque los estudios publicados todavía son escasos y arrojan resultados discordantes. 
A continuación se describen los diferentes tipos de stents:

a. Prótesis tipo Palmaz: aunque inicialmente fueron diseñados para usar en lesiones endovasculares, pronto se convirtieron en los primeros en ser aplicados en bronquios y tráquea. Son prótesis metálicas que vienen colapsadas; se montan en un catéter con balón y se insertan con broncoscopio rígido controlado con visión fluoroscópica. Una vez ubicado en el sitio estrecho, se expande con el balón hasta el diámetro deseado. Existen versiones en forma de Y para ubicar a nivel de la carina a fin de corregir lesiones de tráquea distal y bronquios fuentes para obtener así mejor posicionamiento $\mathrm{y}$ estabilidad.

b. Stents autoexpandibles metálicos: hoy están disponibles en varios modelos, pero dadas las complicaciones reportadas no se usan habitualmente en niños. Los de nitinol (Ultrafex ${ }^{\circledR}$ ) son hechos de titanio, tienen memoria específica y pueden ser usados en niños pues se expanden con la temperatura corporal hasta el diámetro deseado. Los stents metálicos, por su parte, dan buena estabilidad, disminuyen significativamente la limpieza mucociliar o la ventilación colateral, pero tienden a involucrarse en la mucosa; comúnmente desarrollan granulación y causan obstrucción y estenosis residual posterior a su remoción. También se han descrito erosiones de la pared bronquial, perforación vascular y migración a órganos circundantes. En particular al involucrar a la mucosa, los hacen difíciles de retirar y pueden provocar algunos desenlaces fatales, por lo cual se consideran permanentes, si bien son susceptibles de dilatarse a medida que el niño crece.

c. Stents de silicona: son más biocompatibles que los metálicos. El stent Dumon ${ }^{\circledR}$ de silicona se inserta mediante un aplicador específico vía broncoscopio rígido. Están disponibles para lactantes y niños, mediante un introductor pediátrico; sin embargo no permiten la ventilación colateral por tratarse de tubos sellados. No existen todavía en forma de Y para uso pediátrico. Los stents de silicona son fáciles de insertar y remover gracias a su buena buena biocompatibilidad; aun así causan alteración de la depuración mucociliar requiriendo aplicación diaria de aerosoles y de terapia respiratoria. Pueden desarrollarse tapones de moco y requerir nuevas broncoscopias. Tienen mayor tendencia a migrar que los metálicos, de ahí que se requiera vigilancia en centros especializados es caso de complicaciones.

Si bien no existe aún el stent ideal, se hacen intentos por desarrollar nuevos modelos, los cuales mezclan las ventajas de cada uno. Los de nitinol cubierto con poliuretano son mejor tolerados por el epitelio y de fácil remoción, no obstante pueden producir granulomas y reducir la depuración mucociliar.

Existe poca información sobre efectos a largo plazo; estos se dejan como elementos de segunda línea y restringidos a casos seleccionados cuando el manejo convencional ha fracasado. Se espera el desarrollo de stents con cubierta polimérica y materiales bioabsorbibles. Con la mejoría en la biocompatibilidad y estabilidad se podría ampliar su utilidad en la patología bronquial en niños.

\section{Aplicación de medicamentos en la vía aérea}

En varios estudios controlados se ha ensayado el uso de varios tipos de medicamentos en patologías específicas. El medicamento más usado es la dornasa alfa, recomendada para la resolución de atelectasias lobares resistentes al tratamiento médico en particular las producidas por tapones de mocos. La mayor experiencia se reporta en niños con fibrosis quística. En esta enfermedad la instilación de una dosis de dornasa alfa de 2,5 mg diluido en $10 \mathrm{~mL}$ de SSN, vía endoscópica, ha tenido una tasa alta de éxito. También hay 
experiencias satisfactorias en atelectasias, en enfermedades obstructivas crónicas como displasia broncopulmonar, cuadriplejia y estatus asmático.

Otro medicamentos que se han usado en casos específicos son la bleomicina, para manejo de lesiones neoplásicas traqueales, la mitomicina para evitar proliferación tisular post-resección o dilatación de estenosis, el cidofovir para infiltración de papilomatosis traqueobronquial y el ácido tricloroacético para el cierre de fístulas traqueoesofágicas recidivantes. Está en estudio la aplicación de antibióticos tópicos en infecciones localizadas, entre otros.

\section{Extracción de cuerpos extraños}

Las características comportamentales propias de los niños, principalmente en los menores de 4 años, condicionan la aspiración de muchos tipos de cuerpos extraños a la vía aérea. Existen múltiples tipos de objetos que son aspirados por los niños y se pueden alojar en diferentes segmentos de la vía aérea; en particular se han encontrado restos alimentarios (principalmente semillas, maní o cacahuates), objetos metálicos, juguetes, fichas, puntas de lapicero, etc., los cuales se convierten en una fuente importante de morbilidad dada su capacidad de obstruir o dañar la vía aérea. En general, la remoción se lleva a cabo con broncoscopio rígido, ya que es el instrumento que permite un mejor manejo de la vía aérea y da la posibilidad de usar pinzas de tamaño y características más apropiadas para la extracción. La técnica varía según el operador pero siempre se necesita gran experticia para lograr éxito en la extracción con el mínimo daño a la vía aérea. En ocasiones se han logrado extracciones exitosas con fibrobroncoscopios flexibles pero se requiere un equipo de calibre apropiado que permita el paso de instrumental necesario como pinzas, asas o canastillas mediante las cuales se pueden sacar pequeños cuerpos extraños bronquiales. En situaciones complejas se han realizado extracciones con técnicas combinadas en las que el fibrobroncoscopio sirve para el abordaje inicial y la planeación del procedimiento seguido por extracción a través del instrumento rígido.

\section{Aplicaciones potenciales y dirección futura}

Es obvio esperar que muchos avances tecnológicos que han aparecido en adultos puedan ser aplicables en el niño en un futuro no muy lejano. La variada y severa patología respiratoria del adulto ha llevado a avances significativos en el estudio y manejo de estas lesiones, en especial las de carácter maligno, propio de la edad avanzada. Es así como se han desarrollado técnicas endoscópicas muy refinadas que permiten el estudio, incluso hasta un nivel histopatológico, de las lesiones que hay en la vía aérea.

La ecobroncoscopia (EBUS, su sigla en inglés, por endobronchial ultrasound) que mezcla la broncoscopia y la ecografía, permite el estudio de lesiones que están en contacto con la vía aérea (adenomegalias, tumores), que puedan ser biopsiadas desde el lado bronquial, al tiempo que se aprovecha la exactitud y precisión simultáneamente con una ecografía transbronquial que guía al broncoscopista en cuanto al punto exacto de toma de la muestra mediante aguja fina.

Esta técnica es hoy un estándar de diagnóstico en lesiones centrales y periféricas en adultos $\mathrm{y}$ ha permitido tomar decisiones terapéuticas sin recurrir a métodos más invasivos y riesgosos. Se piensa que en un futuro cercano, con el desarrollo de equipos adaptados al tamaño del niño, esta técnica podría ser utilizarse en el estudio de lesiones mediastinales, tales como sospecha de adenomegalias tuberculosas o masas mediastinales como linfomas, etc.

Otra técnica muy utilizada hoy para la localización de lesiones premalignas en el adulto es la autofluorescencia, que aprovecha un principio físico que permite ubicar lesiones neoplásicas en fases iniciales. Mediante este método se puede decidir el tipo de intervención a realizar y adelantarse al avance de la enfermedad neoplásica. 


\section{Conclusiones y recomendaciones}

Los avances tecnológicos, el mejor conocimiento de la patología de la vía aérea y el entrenamiento correcto de los especialistas en neumología pediátrica y en vía aérea, permitirán solucionar de manera eficaz y segura muchos problemas que afectan la vía aérea baja del niño. El trabajo coordinado entre neumólogos, anestesiólogos, intensivistas y cirujanos es indispensable para lograr resultados exitosos que le den funcionalidad y calidad de vida a un importante número de niños enfermos que requieren intervencionismo broncoscópico. De otro lado, la creación de centros especializados en Colombia, que cuenten con tecnología de punta y personal capacitado, son imperativas para cambiar el paradigma de que muchos problemas serios de la vía aérea pediátrica que no tienen solución. Se espera avanzar en ese sentido a fin de ofrecer más oportunidades de mejoría a los niños con enfermedad respiratoria severa en el país.

\section{Lecturas recomendadas}

- Bolliger CT, Mathur PN, Beamis JF, Becker HD, Cavaliere S, Colt H. ERS/ATS statement on interventional pulmonology. European Respiratory Society/American Thoracic Society. Eur Respir J. 2002;19:356.

- $\quad$ Ernst A, Silvestri GA, Johnstone D. Interventional pulmonary procedures: Guidelines from the American College of Chest Physicians. Chest. 2003;123:1693.

- Mehta AC, Harris RJ, De Boer GE. Endoscopic management of benign airway stenosis. Clin Chest Med. 1995;16:401.

- Prakash UB. Advances in bronchoscopic procedures. Chest. 1999;116:1403.

- Cohen MD, Weber TR, Rao CC. Balloon dilation of tracheal and bronchial stenosis. AJR Am J Roentgenol. 1984;142:477.

- Groff DB, Allen JK. Grüentzig balloon catheter dilation for acquired bronchial stenosis in an infant. Ann Thorac Surg. 1985;93:379.

- Fowler CL, Aaland MO, Harris FL. Dilatation of bronchial stenosis with Grüentzig balloon. J Thorac Cardiovasc Surg. 1987;93:308.

- Carlin BW, Harrell II JH, Moser KM. The treatment of endobronchial stenosis using balloon catheter dilation. Chest. 1988;93:1148.
- Nakamura K, Terada N, Ohi M, Matsushita T, Kato N, Nakagawa T. Tuberculous bronchial stenosis: Treatment with balloon bronchoplasty. AJR Am J Roentgenol. 1991; $157: 1187$.

- Hautmann H, Gamarra F, Pfeifer KJ, Huber RM. Fiberoptic bronchoscopic balloon dilatation in malignant tracheobronchial disease: indications and results. Chest. 2001;120:43.

- $\quad$ Noppen M, Schlesser M, Meysman M, D’Haese J, Peche R, Vincken W. Bronchoscopic balloon dilatation in the combined management of postintubation stenosis of the trachea in adults. Chest. 1997;112:1136.

- Girard P, Baldeyron P, Lemoine G, Grunemald D. Left mainstem bronchial stenosis complicating bronchial artery embolization. Chest. 1990;97:1246.

- $\quad$ Ball JB, Delaney JC, Evans CC, Donnelly RJ, Hind CR. Endoscopic bougie and balloon dilatation of multiple bronchial stenoses: 10 year follow up. Thorax. 1991;46:933.

- $\quad$ Keller C, Frost A. Fiber optic bronchoplasty: Description of a simple adjunct technique for the management of bronchial stenosis following lung transplantation. Chest. 1992;102:995.

- Carré P, Rousseau H, Lombart L, Didier A, Dahan M, Fournial $\mathrm{G}$, Léophonte P. Balloon dilatation and self-expanding metal wallstent insertion. For management of bronchostenosis following lung transplantation. The Toulouse Lung Transplantation Group. Chest. 1994;105:343.

- $\quad$ Fouty BW, Pomeranz M, Thigpen TP, Martin RJ. Dilatation of bronchial stenoses due to sarcoidosis using a flexible fiber optic bronchoscope. Chest. 1994;106:677.

- Ferretti G, Jouvan FB, Thony F, Pison C, Coulomb M. Benign noninflammatory bronchial stenosis: Treatment with balloon dilation. Radiology. 1995;196:831.

- Mayse ML, Greenheck J, Friedman M, Kovitz KL. Successful bronchoscopic balloon dilation of nonmalignant tracheobronchial obstruction without fluoroscopy. Chest. 2004;126:634.

- Vansteenkiste JF, Lacquet LM. Possibilities and indications for Nd-YAG laser and dilation therapy in the management of tracheal stenosis. Acta Otorhinolaryngol Belg. 1995;49:359.

- Brown SB, Hedlund GL, Glasier CM, Williams KD, Greenwood LH, Gilliland JD. Tracheobronchial stenosis in infants: successful balloon dilation therapy. Radiology. 1987;164:475.

- Wilson NJ. Bronchoscopic observations in tuberculous tracheobronchitis: Clinical and pathological correlation. Dis Chest. 1945;11:36.

- $\quad$ Bugher JC, Littig J, Culp J. Tuberculous tracheobronchitis: Its pathogenesis. Am J Med Sci. 1937;193:515.

- Auerbach O. Tuberculosis of the trachea and major bronchi. Am Rev Tuberc. 1949;60:604.

- Eloesser L. Bronchial stenosis in pulmonary tuberculosis with some notes on tuberculous stenosis of the trachea and the bronchioles. Am Rev Tuberc. 1934;30:123. 
- Salkin D, Cadden AV, Edson RC. The natural history of tuberculous tracheobronchitis. Am Rev Tuberc. 1943;47:351.

- Judd AR. Tuberculous tracheobronchitis: A study of 500 consecutive cases. J Thorac Surg. 1947;16:512.

- Dumon JF. YAG Laser bronchoscopy. Praeger Publishers, New York, 1985. p. 117.

- Elkerbout SC, van Lingen RA, Gerriten J, Roorda RJ. Endoscopic balloon dilatation of acquired airway stenosis in newborn infants: A promising treatment. Arch Dis Child. $1993 ; 68: 37$

- Hebra A, Powell DD, Smith CD, Othersen Jr HB. Balloon tracheoplasty in children: Results of a 15 year experience. J Pediatr Surg. 1991;26:957.

- Sheski FD, Mathur PN. Long-term results of fiberoptic bronchoscopic balloon dilation in the management of benign tracheobronchial stenosis. Chest. 1998;114:796.

- Grillo HC, Mathisen DJ. Surgical management of tracheal stricture. Surg Clin North Am. 1988; 68:511.

- Iles, PB. Multiple bronchial stenoses: Treatment by mechanical dilatation. Thorax. 1981;36:784.

- Mehta AC, Lee FY, Cordasco EM, Kirby T, Eliachar I, De Boer G. Concentric tracheal and subglottic stenosis. Chest. 1993;104:674.

- Dumon JF, Rebound D, Garbe L, et al. Treatment of tracheobronchial lesions by laser photo resection. Chest. 1982;81:278.

- Mathur PN, Wolf KF, Busk MD, et al. A fiberoptic bronchoscopic cryotherapy in the management of tracheobronchial obstruction. Chest. 1996;110:718. 